九州大学学術情報リポジトリ

Kyushu University Institutional Repository

An advanced switching parameter for a hybrid LES/RANS model considering the characteristics of near-wall turbulent length scales

ABE, Ken-ichi

Department of Aeronautics and Astronautics, Faculty of Engineering, Kyushu University : Professor

http://hdl. handle. net/2324/4150511

出版情報: Theoretical and Computational Fluid Dynamics. 28 (5)，pp.499-519，2014-08-09. Springer nature

バージョン :

権利関係 : 


\title{
An advanced switching parameter for a hybrid LES/RANS model considering the characteristics of near-wall turbulent length scales
}

\author{
Ken-ichi ABE
}

Received: date / Accepted: date

\begin{abstract}
In this study, we proposed an idea for an advanced switching parameter used in a hybrid approach connecting large eddy simulation (LES) with Reynolds-averaged Navier-Stokes (RANS) modeling (the hybrid LES/RANS (HLR) model). Although the HLR model is promising way to predict engineering turbulent flows, an important problem is that RANS is always adopted in the near-wall region, even if the grid resolution is fine enough for LES. To overcome this difficulty, the switching parameter proposed here introduced knowledge of the Kolmogorov microscale that is thought to be reasonable for representing the near-wall turbulence. This parameter enabled the present HLR model to be smoothly replaced by a full LES if a grid resolution was fine enough in the near-wall region. To confirm model performance, the present HLR model was applied to numerical simulations of a periodic hill flow as well as fundamental plane channel flows. The model generally provided reasonable predictions for these test cases that include complex turbulence with massive flow separation.
\end{abstract}

Keywords Turbulence · Large Eddy Simulation · Hybrid LES/RANS Model · Near-wall Turbulent Structures · Kolmogorov Microscale

\section{Introduction}

Large eddy simulation (LES) needs far fewer grid nodes compared with direct numerical simulation (DNS) because only the grid-scale (GS) eddies are to be directly resolved, while the subgrid-scale (SGS) eddies are modeled. Thus far, a significant number of research groups have proposed several kinds of SGS

Ken-ichi ABE

Professor, Department of Aeronautics and Astronautics, Kyushu University, 744 Motooka, Nishi-ku, Fukuoka 819-0395, Japan

Tel.: $+81-92-8023023$

Fax: +81-92-8023001

E-mail: abe@aero.kyushu-u.ac.jp 
models (see for example, [1]-[7]). Although LES is known to be a useful way to predict complex turbulence in engineering applications, there still remains a serious difficulty in its application to very high Reynolds-number $(R e)$ flows. For instance, reasonable LES for a channel flow needs a grid resolution such as $\Delta x^{+} \sim 100$ ( $x$ : the streamwise direction) and $\Delta z^{+} \sim 20$ ( $z$ : the spanwise direction) in the near-wall region, as well as $\Delta y^{+} \sim 1$ in the wall-normal direction, where the no-slip condition is specified at the wall surface. Note that ()$^{+}$denotes a value normalized by the friction velocity $u_{\tau}$. When LES is applied to the channel flow at $R e_{\tau}=10^{4}$, where $\operatorname{Re}_{\tau}\left(=u_{\tau} \delta / \nu\right)$ is the Reynolds number based on the half channel height $(\delta)$, the kinematic viscosity $(\nu)$ and the friction velocity, the aforementioned grid resolution $\Delta z^{+}=20$ translates into $\Delta z=2 \times 10^{-3} \delta$ (i.e., 500 grid points per $\delta$ in $z$-direction). Such a grid-resolution requirement for high-Re LESs is significant, even if it is applied to simple wall-shear flows.

To overcome this difficulty, one promising approach is the so-called "hybrid LES/RANS (HLR) model." This model was originally based on the concept of a hybrid model connecting LES with Reynolds-averaged Navier-Stokes (RANS) modeling in the near-wall region. Thus far, a number of research groups have addressed this challenging problem (see for example, [8]-[19]). A representative model of this type of approach is the "detached eddy simulation (DES)" (Nikitin et al.[9]). DES was studied very actively by many research groups, among which an advanced version of DES, called the "delayed detached eddy simulation (DDES)," was proposed to improve model performance (see for example, Spalart et al.[17]). On the other hand, Breuer et al.[18] developed a non-zonal HLR model based on a one-equation nearwall model. Aside from these non-zonal HLR models, Deck[19] discussed the capabilities of a recently-developed zonal type of HLR model. Although most previous HLR models adopted a linear eddy-viscosity form for representing the SGS and Reynolds stresses, Abe[16] proposed an HLR model that introduced a non-linear eddy-viscosity model (NLEVM) to resolve the near-wall stress anisotropy more correctly. Each study provided encouraging results and useful insights into developing HLR modeling. Nevertheless, there still remains an important issue: in general, such an HLR model always adopts a RANS model near the wall surface.

Originally, the HLR model was a turbulence model that used LES in the region far from the wall; the modeling was then connected with RANS in the near-wall region. A strategy to connect these LES and RANS regions smoothly may be the following hybrid approach (see for example, Abe[16]):

$$
\phi=\left(1-f_{h b}\right) \phi_{(R A N S)}+f_{h b} \phi_{(L E S)},
$$

where $\phi$ is a flow variable and $f_{h b}$ is a switching function. In Eq. (1), $f_{h b}$ is close to 1 in the region far from the wall, where LES is adopted. Conversely, as $f_{h b}$ approaches 0 , the RANS calculation is adopted. For instance, the following switching function is used in [16]:

$$
f_{h b}=1-\exp \left\{-\left(\alpha \frac{y}{\Delta}\right)^{\beta}\right\},
$$




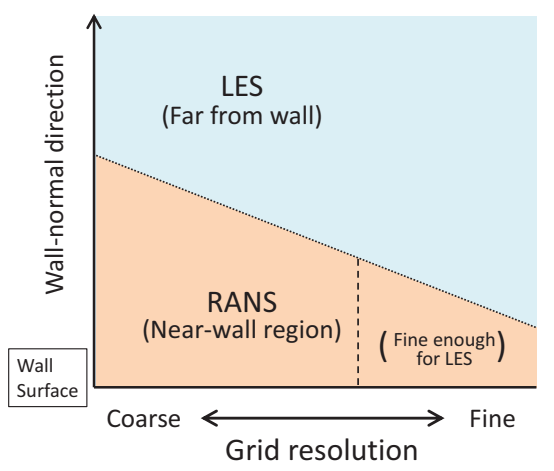

(a) Conventional hybrid model

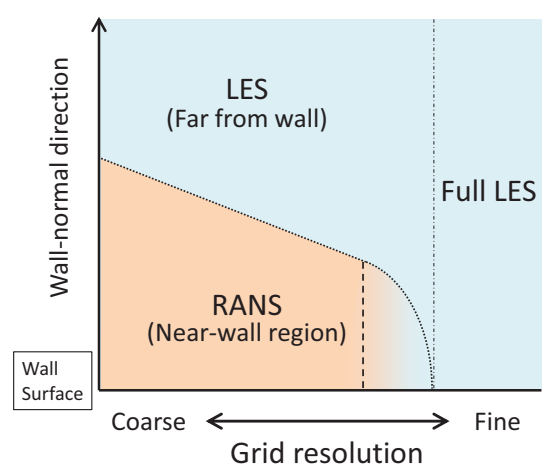

(b) Advanced hybrid model

Fig. 1 Domain decomposition in a hybrid LES/RANS model.

where $\alpha$ and $\beta$ are model constants, and $\Delta$ is an SGS filter width. Considering Eq. (2), LES is applied in the region where the grid resolution is fine enough to guarantee $\Delta<\alpha y$. This function is expected to effectively work for cases with rather coarse grid resolutions, while the strict "full LES" is never applied even if the grid resolution is fairly fine. Since $y$ approaches zero toward the wall surface, there inevitably exists a region of $\alpha y<\Delta$. Although alternative approaches introducing turbulence variables may be available instead of Eq. (2), RANS has always been adopted in the near-wall region in previous HLR models.

To facilitate an understanding of this issue, Fig. 1 illustrates the basic concept of the domain decomposition in an HLR model. Figure 1 (a) shows the domain-decomposition pattern in most conventional HLR models. However, it is of course preferable to apply LES to the entire flow domain under a sufficiently fine grid-resolution condition. To realize this requirement, the domaindecomposition pattern must be specified as shown in Fig. 1 (b). Therefore, in order to adopt either LES or RANS more properly depending on the near-wall grid resolution, a new idea for the switching function $f_{h b}$ may be necessary.

Based on the background provided above, the primary goal of the present study is to propose a new switching parameter for a HLR model to realize Fig. 1 (b). This switching parameter introduces knowledge of the Kolmogorov microscale that is thought to be reasonable for representing the near-wall turbulence. By using this parameter, an HLR model is expected to be smoothly replaced by a full LES if a grid resolution is fine enough in the near-wall region. Furthermore, another important concern of an HLR model is how wide a range can be covered by LES. For this purpose, the anisotropy-resolving SGS model proposed by Abe[20] may be effective in the LES region. In this model, the SGS-stress expression is constructed by combining an isotropic eddy-viscosity model (EVM) with an extra anisotropic term. Applying the model to fundamental test cases indicated the basic capability of this SGS modeling concept[20][21]. In this study, we construct a new advanced one- 
equation HLR model adopting both this new switching parameter in $f_{h b}$ and the anisotropic SGS model[20] in the LES region. To assess the basic performance of this model, numerical simulations are performed for a periodic hill flow as well as fundamental plane channel flows. The results that we obtained are investigated in detail for elucidating the usefulness of the present HLR model for engineering applications.

\section{Turbulence Model}

\subsection{Governing equations}

The filtered (or Reynolds-averaged) governing equations for an incompressible turbulent flow may be written as

$$
\begin{gathered}
\frac{\partial \bar{U}_{i}}{\partial x_{i}}=0 \\
\frac{\mathrm{D} \bar{U}_{i}}{\mathrm{D} t}=-\frac{1}{\rho} \frac{\partial \bar{P}}{\partial x_{i}}+\frac{\partial}{\partial x_{j}}\left\{\nu\left(\frac{\partial \bar{U}_{i}}{\partial x_{j}}+\frac{\partial \bar{U}_{j}}{\partial x_{i}}\right)-\tau_{i j}\right\},
\end{gathered}
$$

where $\overline{(~}$ ) denotes a filtered value in the LES region or a Reynolds-averaged value in the RANS region, respectively. In Eq. (4), $\rho, \bar{P}$ and $\bar{U}_{i}$ denote the density, filtered static pressure and filtered velocity, respectively. The SGS stress $\tau_{i j}$ in the LES region is originally expressed as

$$
\tau_{i j}=\overline{U_{i} U_{j}}-\bar{U}_{i} \bar{U}_{j} .
$$

Note that $\tau_{i j}$ coincides with the following general expression for the Reynoldsstress tensor in the RANS region:

$$
\tau_{i j}=\overline{u_{i} u_{j}},
$$

where $u_{i}$ is defined as $u_{i}=U_{i}-\bar{U}_{i}$.

\subsection{An anisotropy-resolving SGS model in the LES region}

We now briefly describe the anisotropy-resolving SGS model[20], which is adopted in the LES region. The SGS stress $\tau_{i j}$ in Eq. (4) is modeled as follows:

$$
\tau_{i j}=\frac{2}{3} k_{S G S} \delta_{i j}-2 \nu_{S G S} S_{i j}+2 k_{S G S} b_{i j}^{E A T},
$$

where $k_{S G S}$ and $\nu_{S G S}$ are the SGS turbulence energy and the SGS eddy viscosity, respectively. The strain-rate tensor $S_{i j}$ is expressed as

$$
S_{i j}=\frac{1}{2}\left(\frac{\partial \bar{U}_{i}}{\partial x_{j}}+\frac{\partial \bar{U}_{j}}{\partial x_{i}}\right) .
$$


In Eq. (7), the anisotropy tensor $b_{i j}^{E A T}$ in the extra anisotropic term on the right-hand side is modeled as

$$
b_{i j}^{E A T}=\frac{\tau_{i j}^{\prime}-\left(-2 \nu^{\prime} S_{i j}\right)}{\tau_{k k}^{\prime}-\left(-2 \nu^{\prime} S_{k k}\right)}-\frac{1}{3} \delta_{i j}=\frac{R_{i j}^{\prime}}{\tau_{k k}^{\prime}}, \quad R_{i j}^{\prime}=\tau_{i j}^{\prime a}-\left(-2 \nu^{\prime} S_{i j}\right),
$$

where $\tau_{i j}^{\prime{ }^{a}}=\tau_{i j}^{\prime}-\tau_{k k}^{\prime} \delta_{i j} / 3$.

In Eq. (9), $\tau_{i j}^{\prime}$ is given by the following representative scale-similarity model of Bardina et al.[2]:

$$
\tau_{i j}^{\prime}=\left(\bar{U}_{i}-\widehat{\bar{U}}_{i}\right)\left(\bar{U}_{j}-\widehat{\bar{U}}_{j}\right)
$$

where $\widehat{(~)}$ denotes a test-filtered value. In the model, $\nu^{\prime}$ is an equivalent eddy viscosity evaluated by an EVM-type linear approximation for Eq. (10) as

$$
\tau_{i j}^{\prime a} S_{i j}=-2 \nu^{\prime} S_{i j} S_{i j}=-2 \nu^{\prime} S^{2} \quad \longrightarrow \quad \nu^{\prime}=-\frac{\tau_{i j}^{\prime}{ }^{a} S_{i j}}{2 S^{2}},
$$

where $S^{2}=S_{i j} S_{i j}$. Therefore, $R_{i j}^{\prime}$ is evaluated by subtracting an EVM form from the original Bardina et al.[2] model. Considering the fact that the production term of $k_{S G S}$ is expressed as $-\tau_{i j}^{\prime} \bar{U}_{i, j}\left(=-\tau_{i j}^{\prime} S_{i j}\right)$, Eq. (11) means that this linearized approximation produces the same amount of energy transfer between the GS and SGS components as the original scale-similarity model. Therefore, $R_{i j}^{\prime}$ yields no undesirable extra energy transfer between the GS and SGS components; the extra term in Eq. (7) is then expected to successfully predict the SGS-stress anisotropy with no serious effects on computational stability.

Concerning the linear EVM in Eq. (7), we basically adopts the one-equation SGS model proposed by Inagaki[22] with some minor modifications. The SGS viscosity $\nu_{S G S}$ is modeled as follows:

$$
\begin{aligned}
& \nu_{S G S}=C_{S G S} f_{S G S} \sqrt{k_{S G S}} \Delta, \quad f_{S G S}=1-\exp \left\{-\left(\frac{y_{\varepsilon}^{\prime}}{A_{0}}\right)^{4 / 3}\right\}, \\
& y_{\varepsilon}^{\prime}=\left\{\frac{\left(\nu \varepsilon_{S G S}\right)^{1 / 4} y}{\nu}\right\} \sqrt{C_{l} \frac{y}{\Delta}}, \quad C_{S G S}=0.05, \quad A_{0}=30, \quad C_{l}=4,
\end{aligned}
$$

where $\varepsilon_{S G S}$ is the dissipation rate for $k_{S G S}$. In this study, $k_{S G S}$ is evaluated using the following equations:

$$
\begin{gathered}
\frac{\mathrm{D} k_{S G S}}{\mathrm{D} t}=\frac{\partial}{\partial x_{j}}\left\{\left(\nu+\frac{\nu_{S G S}}{\sigma_{S G S}}\right) \frac{\partial k_{S G S}}{\partial x_{j}}\right\}-\tau_{i j} \frac{\partial \bar{U}_{i}}{\partial x_{j}}-\varepsilon_{S G S}, \\
\varepsilon_{S G S}=C_{\varepsilon} \frac{k_{S G S}{ }^{3 / 2}}{\Delta}+\frac{2 \nu k_{S G S}}{y^{2}} .
\end{gathered}
$$

The model constants are specified as $\sigma_{S G S}=0.5$ and $C_{\varepsilon}=0.835$ (Inagaki[22]). 
According to [20], the SGS filter width $\Delta$ is defined as follows:

$$
\Delta=\sqrt{\text { the maximum area among the faces of a cell }} .
$$

In the Cartesian structured grid system, the above definition is rewritten as

$$
\Delta=\sqrt{\max \left(\Delta_{x} \Delta_{y}, \Delta_{y} \Delta_{z}, \Delta_{z} \Delta_{x}\right)} .
$$

This definition is the same as that given in the HLR model in [16]. Note that by using Eq. (15), we can reasonably determine $\Delta$ even in a non-orthogonal or an unstructured grid system. More detailed descriptions of the present anisotropyresolving SGS model are given in [20].

\subsection{A one-equation NLEVM in the RANS region}

To connect the LES and RANS regions more smoothly, we develop a new oneequation NLEVM that is applied in the RANS region. The basic formulation of the present model is the same as that used in [16]. This model was originally proposed by Abe et al.[23] to reproduce the near-wall turbulence more correctly. Based on this two-equation NLEVM, we attempt to model the dissipation rate $\varepsilon$ algebraically, instead of solving its transport equation. Similar to the model of Breuer et al.[18], both the SGS and RANS models used in the present HLR model belong to the same category of one-equation turbulence models. Therefore, these two models are expected to connect smoothly between the LES and RANS regions because the same type of wall-boundary conditions can be applied for all flow variables.

For this purpose, we consider an algebraic model to represent the wallshear turbulence. It is well known that an equilibrium boundary layer exhibits the following relation for the local equilibrium condition in the logarithmic region:

$$
\frac{k^{3 / 2}}{\varepsilon} \sim\left(C_{\mu}^{-3 / 4} \kappa\right) y \sim C_{e q} y \quad \longrightarrow \quad \varepsilon \sim \frac{k^{3 / 2}}{C_{e q} y},
$$

where $k\left(=\overline{u_{i} u_{i}} / 2\right)$ is the turbulence energy. In Eq. (17), the conventional values of $C_{\mu}(=0.09)$ and $\kappa(=0.41)$ result in $C_{e q} \sim 2.5$. On the other hand, in the vicinity of the wall, the wall-limiting behavior of turbulence yields the relation $\varepsilon=2 \nu(\partial \sqrt{k} / \partial y)^{2}$ or $\varepsilon=2 \nu k / y^{2}$. Taking into account this knowledge, we propose the following algebraic model for $\varepsilon$ :

$$
\varepsilon=f_{e q} \frac{k^{3 / 2}}{C_{e q} y}+\frac{2 \nu k}{y^{2}},
$$

where $f_{e q}$ is a damping function introduced to adjust the distribution of $\varepsilon$. In this study, the following model is used simply to suppress the excessive peak of the first term on the right-hand side in Eq. (18):

$$
f_{e q}=1-\exp \left\{-\left(\frac{\sqrt{k} y}{50 \nu}\right)^{2}\right\} \text {. }
$$




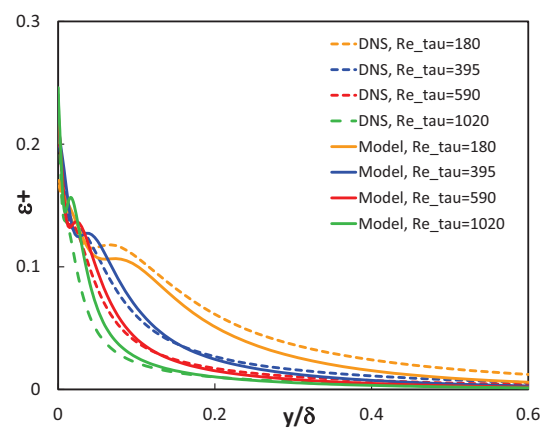

(a) Global range

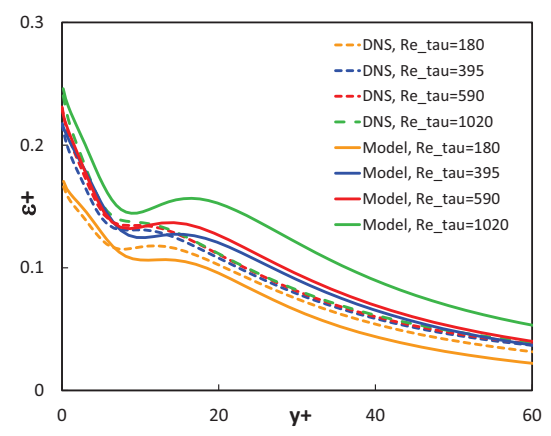

(b) Near-wall region

Fig. 2 A priori test of the algebraic dissipation-rate model using the DNS data.

To confirm the basic performance of Eq. (18), Fig. 2 compares the results of an a priori test using the DNS data with the original true values. Note that the DNS data for $R e_{\tau}=395$ and 590 were taken from Moser et al.[24] and those for $R e_{\tau}=180$ and 1020 from Abe et al.[25]. As seen in the figure, this algebraic model generally provides reasonable predictions, at least for the test cases of attached wall-shear turbulence, although small discrepancies are still seen between the predictions and the DNS data.

In this study, the turbulence energy is determined from the typical form of the transport equation:

$$
\frac{\mathrm{D} k}{\mathrm{D} t}=\frac{\partial}{\partial x_{j}}\left\{\left(\nu+\frac{\nu_{t}}{\sigma_{k}}\right) \frac{\partial k}{\partial x_{j}}\right\}-\overline{u_{i} u_{j}} \frac{\partial \bar{U}_{i}}{\partial x_{j}}-\varepsilon,
$$

where $\nu_{t}$ is the eddy viscosity and $\sigma_{k}(=1.2)$ is the model constant. The other model expressions are described in the Appendix. We note again that the present model expressions for $k$ and $\varepsilon$ (i.e., Eqs. (20) and (18), respectively) look similar to those seen in Eqs. (13) and (14), respectively.

\subsection{A new switching parameter for an advanced HLR model}

To realize the requirement shown in Fig. 1 (b), we propose a new switching strategy between the LES and RANS regions. As described above, the HLR is a turbulence model that uses LES in the region far from the wall, and then connects with RANS in the near-wall region. Although we also adopt the hybrid approach expressed in Eq. (1), some important modifications are newly introduced in this study. Since the present HLR model adopts the same type of one-equation turbulence models for both the LES and RANS regions, only the following variables should be connected by means of Eq. (1):

- the SGS-stress tensor $\left(\tau_{i j}\right)$ in Eq. (4)

- the dissipation rate in Eqs. (14) and (18) 
- the eddy viscosity and the model coefficient for the turbulent diffusion in Eqs. (13) and (20)

We can understand again from Eq. (2) that a conventional HLR model always adopts a RANS model in the region close to the wall surface. For instance, the following switching function for $f_{h b}$ is used in [16]:

$$
f_{h b}=1-\exp \left\{-\left(\frac{y}{4.0 \Delta}\right)^{6}\right\} .
$$

This equation means that the strict "full LES" is never applied even if the grid resolution is fairly fine. To apply LES to the entire flow domain under a sufficiently fine grid resolution, a new idea for the switching function $f_{h b}$ may be necessary. In this sense, an advantage of the present model is that the same boundary conditions can be applied for both LES and RANS at the wall surface. This feature makes us never mind the boundary conditions that may possibly change according to the value of $f_{h b}$ ranging from 0 to 1 .

Considering these factors, we propose a new alternative length-scale model instead of the wall distance $y$ used in Eq. (2):

$$
l_{K L}=\frac{1}{C_{K L}^{3 / 2} f_{K L}} \frac{k^{3 / 2}}{\varepsilon},
$$

where $C_{K L}(=15)$ is the model constant and $f_{K L}$ is the model function as described below. This model is originally based on the length scale of an energycontaining eddy with some modifications in the near-wall region. In fact, the following function $f_{K L}$ in Eq. (22) is modeled to represent the characteristics of the near-wall turbulence:

$$
f_{K L}=1-\exp \left\{-\left(\frac{\sqrt{k} y}{C_{K L} \nu}\right)^{3 / 2}\right\} .
$$

Considering the near-wall limiting behavior of turbulence, the following estimation for the length scale can be obtained from Eqs. (22), (23) and (18) as the wall approaches:

$$
\begin{gathered}
l_{K L} \sim \frac{1}{C_{K L}^{3 / 2}\left(\sqrt{k} y / C_{K L} \nu\right)^{3 / 2}} \frac{k^{3 / 2}}{2 \nu k / y^{2}}=\left(\frac{\nu}{\sqrt{k} y}\right)^{3 / 2} \frac{\sqrt{k} y^{2}}{2 \nu} \\
=\frac{\nu^{1 / 2} y^{1 / 2}}{2 k^{1 / 4}}=\frac{\nu^{3 / 4}}{2^{3 / 4}} \frac{y^{1 / 2}}{2^{1 / 4} \nu^{1 / 4} k^{1 / 4}}=\frac{\nu^{3 / 4}}{2^{3 / 4}}\left(\frac{y^{2}}{2 \nu k}\right)^{1 / 4} \\
=\frac{\nu^{3 / 4}}{2^{3 / 4}} \frac{1}{\varepsilon^{1 / 4}}=\frac{1}{2^{3 / 4}}\left(\frac{\nu^{3}}{\varepsilon}\right)^{1 / 4} .
\end{gathered}
$$

As found in Eq. (24), $l_{K L}$ results in the Kolmogorov microscale that is thought to be reasonable for representing the near-wall turbulence. Note that such a modeling strategy has often been adopted in some low-Re $k-\varepsilon$ models (see 


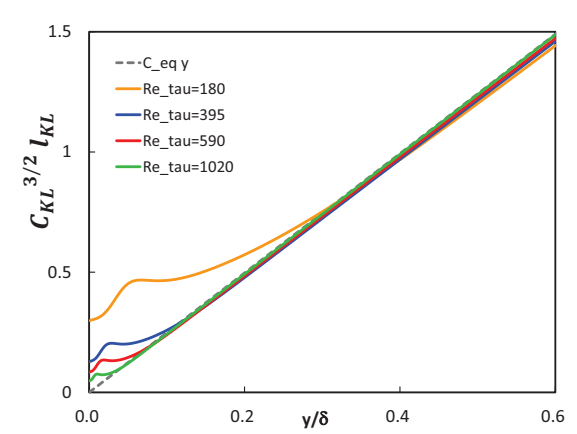

(a) Global distributions

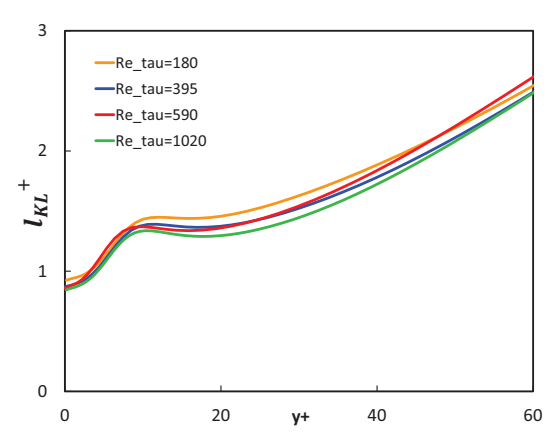

(b) Near-wall distributions in wall units

Fig. 3 A priori test of the present switching length-scale parameter.

for example, Nagano \& Tagawa[26]; Abe et al.[27]). This length scale can be rewritten in wall units as follows:

$$
l_{K L}^{+}=\frac{l_{K L} u_{\tau}}{\nu} \sim \frac{1}{2^{3 / 4}}\left(\frac{\nu^{3}}{\varepsilon}\right)^{1 / 4} \frac{u_{\tau}}{\nu}=\left(\frac{u_{\tau}^{4}}{8 \nu \varepsilon}\right)^{1 / 4}=\frac{1}{\left(8 \varepsilon^{+}\right)^{1 / 4}} .
$$

It is known that the value of $\varepsilon^{+}$is around 0.2 (roughly from 0.1 to 0.25 ) in the near-wall region of a equilibrium boundary layer. Considering this fact, $l_{K L}^{+}$is estimated as $l_{K L}^{+} \sim 0.9$ (roughly from 0.8 to 1.0 ).

On the other hand, since $f_{K L}$ approaches 1 in the region far from the wall, we find from Eq. (17) that this length-scale model returns the following relation:

$$
l_{K L} \sim \frac{1}{C_{K L}^{3 / 2}} \frac{k^{3 / 2}}{\varepsilon} \longrightarrow l_{K L} \sim \frac{C_{e q} y}{C_{K L}^{3 / 2}} \longrightarrow C_{K L}^{3 / 2} l_{K L} \sim C_{e q} y
$$

Equation (26) means that $l_{K L}$ is proportional to the wall distance $y$ in the region far from the wall. To investigate the fundamental features of the present length scale model by Eq. (22), Fig. 3 compares the distributions of $l_{K L}$ obtained by an a priori test using the DNS data for the aforementioned different Reynolds numbers[24][25]. Note that the distribution of $\left(C_{e q} y\right)$ is also included in Fig. 3 (a) for comparison. Since the distribution of $l_{K L}$ is nearly proportional to the wall distance $y$ - except for the near-wall region -, we can successfully use this length scale as a proxy for the wall distance. Conversely, in the near-wall region, $l_{K L}$ does not approach zero but maintains a finite value. An important feature is that the minimum level of $l_{K L}$ decreases as $R e$ increases, which results in the Kolmogorov microscale that depends on $R e$. However, it is interesting that Fig. 3 (b) shows a similar value of $l_{K L}^{+}$ expressed in wall units for all Re cases. This fact accounts for the near-wall limiting behavior described in Eq. (25). 
Taking into account these features of the length scale $l_{K L}$, we propose the following new switching function for the present HLR model:

$$
f_{h b}=1-\exp \left\{-\left(C_{h b} \frac{l_{K L}}{\Delta}\right)^{3}\right\},
$$

where $C_{h b}(=30)$ is the model constant. As discussed above, $l_{K L}$ approaches the Kolmogorov microscale in the vicinity of the wall surface. Therefore, Eq. (27) indicates that the RANS model has no effect unless the near-wall grid width becomes much larger than the Kolmogorov microscale (i.e., $\Delta \gg$ $\left.\left(\nu^{3} / \varepsilon\right)^{1 / 4}\right)$. In other words, if a grid resolution is fine enough to guarantee $\Delta<C_{h b} l_{K L}$, LES can be applied to the entire flow domain because $f_{h b}$ is close to 1 everywhere. Considering the fact that $l_{K L}^{+}$is approximately 0.9 in the near-wall region of wall-shear turbulence, this condition is roughly estimated, in wall units, to be $\Delta^{+}<C_{h b} l_{K L}^{+} \sim 27$. Therefore, the present HLR model guarantees a full LES under a sufficiently fine grid-resolution condition, as shown in Fig. 1 (b). This is a notable feature of the present HLR model that distinguishes it from other previous models.

We now consider a conventional form of the switching function simply using the wall distance $y$ for comparison. The relation of Eq. (26) indicates that the following conventional form of $f_{h b}$ corresponds to Eq. (27), except for the region close to the wall:

$$
f_{h b}=1-\exp \left\{-\left(C_{h b} \frac{C_{e q}}{C_{K L}^{3 / 2}} \frac{y}{\Delta}\right)^{3}\right\} .
$$

Figure 4 compares the distributions of the switching functions predicted by Eqs. (27) and (28) for various grid resolutions. Note that these values are evaluated by an a priori test using the DNS data[24]. We confirm from the broken lines in Fig. 4 that the conventional function Eq. (28) becomes zero as the wall is approached. Even if the grid resolution is sufficiently fine (as $\Delta^{+}=10$ ), RANS is applied in the near-wall region. In contrast, the present switching function - Eq. (27) - returns values around 1 for the entire flow domain in the case of $\Delta^{+}=10$. This behavior is considered to be preferable because such a grid resolution is fine enough for LES. Conversely, in the case of $\Delta^{+}=200$, the new function Eq. (27) yields almost the same distribution as the conventional function given by Eq. (28). This result is also reasonable because the predictive performance of LES tends to worsen with such a coarse grid resolution.

A notable feature may be the distribution of Eq. (27) for $\Delta^{+}=50$, where $f_{h b}$ shows a value between 0 and 1 at the wall surface. Note that a slightly wavy distribution is seen in the near-wall region particularly for $\Delta^{+}=50$. This effect is caused by the characteristics of the length-scale profile as shown in Fig. 3. A non-zero value of $f_{h b}$ at the wall surface is not always preferable for a conventional HLR model because it is generally difficult to specify the boundary conditions of the turbulent variables. In contrast, the present HLR 


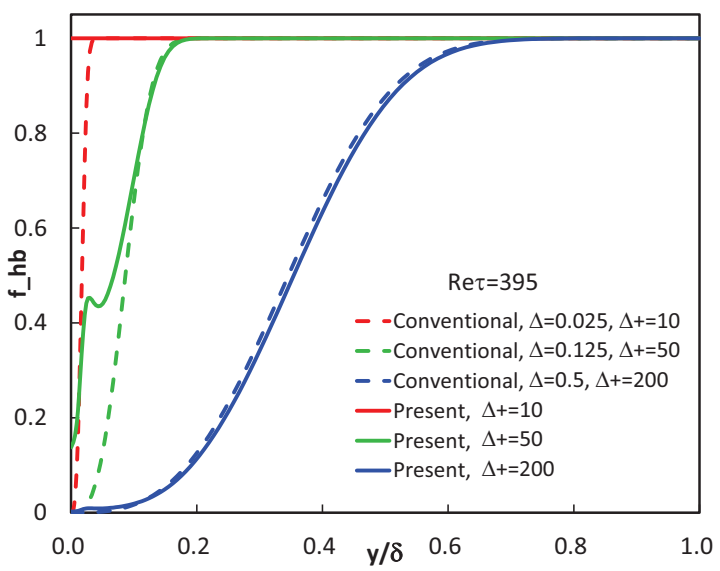

Fig. 4 A priori test for the present switching function $f_{h b}$ defined by Eq. (27) $\left(R e_{\tau}=395\right)$.

model adopts the same type of one-equation turbulence models for both LES and RANS. Since we can use the same boundary conditions for the turbulence energy and its dissipation rate, we do not need to concern ourselves with this issue.

Finally, we investigate the switching location between the LES and RANS regions in more detail. Considering the values of the model constants $\left(C_{h b}=\right.$ 30, $C_{e q} \sim 2.5$, and $\left.C_{K L}=15\right)$, Eq. (28) can be estimated as follows:

$$
f_{h b} \sim 1-\exp \left\{-\left(\frac{y}{0.77 \Delta}\right)^{3}\right\} .
$$

Comparing the constant 0.77 in Eq. (29) with the constant 4.0 in Eq. (21) (Abe[16]), one can understand that LES covers a much wider flow-domain range if the present switching function is adopted. This fact is the second notable feature of the present HLR model. In fact, the most valuable contribution for this is achieved by adopting an advanced anisotropy-resolving SGS model in the LES region[20][21].

\section{Test Cases and Computational Conditions}

First, the present HLR model was applied to fully-developed plane channel flows with various grid resolutions and at various Reynolds numbers. The computational parameters are summarized in Table 1. The Reynolds number $R e_{\tau}\left(=u_{\tau} \delta / \nu\right)$ ranged from 395 to 5000 . We selected the lower-Re cases $\left(R e_{\tau}=395\right)$ for a detailed discussion of the model performance. On the other hand, the higher- $R e$ cases $\left(R e_{\tau}=1020,5000\right)$ provided useful knowledge related to performance when applied to high- $R e$ wall-shear turbulent flows. Note that the $R e$ in C1E3F corresponds to the DNS in Abe et al.[25]. 
Table 1 Computational parameters for channel-flow cases.

\begin{tabular}{lcccccc}
\hline \hline Case & Grid numbers & Domain $(x-z)$ & $\Delta x$ & $\Delta y$ & $\Delta z$ & $\Delta$ \\
\hline C395A & $65 \times 98 \times 65$ & $6.4 \delta \times 3.2 \delta$ & 0.1 & $1 \times 10^{-3}-0.05$ & 0.05 & 0.071 \\
C395B & $33 \times 98 \times 33$ & $6.4 \delta \times 3.2 \delta$ & 0.2 & $1 \times 10^{-3}-0.05$ & 0.1 & 0.14 \\
C395C & $17 \times 98 \times 17$ & $6.4 \delta \times 3.2 \delta$ & 0.4 & $1 \times 10^{-3}-0.05$ & 0.2 & 0.28 \\
C395D & $17 \times 98 \times 17$ & $9.6 \delta \times 4.8 \delta$ & 0.6 & $1 \times 10^{-3}-0.05$ & 0.3 & 0.42 \\
C395E & $17 \times 98 \times 17$ & $16 \delta \times 8 \delta$ & 1 & $1 \times 10^{-3}-0.05$ & 0.5 & 0.71 \\
C1E3F & $33 \times 121 \times 33$ & $8 \delta \times 4 \delta$ & 0.25 & $5 \times 10^{-4}-0.05$ & 0.125 & 0.18 \\
C5E3G & $97 \times 131 \times 49$ & $6.4 \delta \times 1.6 \delta$ & 0.067 & $1.5 \times 10^{-4}-0.05$ & 0.033 & 0.047 \\
\hline \hline
\end{tabular}

\begin{tabular}{lcccccc}
\hline \hline Case & $R e_{\tau}$ & $\Delta x^{+}$ & $\Delta y^{+}$ & $\Delta z^{+}$ & $\Delta^{+}$ & $\Delta t^{+}$ \\
\hline C395A & 395 & 40 & $0.4-20$ & 20 & 28 & $1 \times 10^{-3}$ \\
C395B & 395 & 79 & $0.4-20$ & 40 & 55 & $1 \times 10^{-3}$ \\
C395C & 395 & 158 & $0.4-20$ & 79 & 110 & $1 \times 10^{-3}$ \\
C395D & 395 & 237 & $0.4-20$ & 119 & 166 & $1 \times 10^{-3}$ \\
C395E & 395 & 395 & $0.4-20$ & 198 & 280 & $1 \times 10^{-3}$ \\
C1E3F & 1020 & 250 & $0.5-50$ & 125 & 180 & $5 \times 10^{-4}$ \\
C5E3G & 5000 & 333 & $0.75-250$ & 167 & 235 & $2 \times 10^{-4}$ \\
\hline \hline
\end{tabular}

To investigate the basic performance of the present HLR model, five grid resolutions were selected for cases at $R e_{\tau}=395$. Abe[20] showed that the present anisotropy-resolving SGS model returned generally acceptable predictions for the mean velocity, even for the coarser grid-resolution case of C395C (as well as C395A and C395B). However, it was also reported that there remained slight discrepancies in the distributions of the turbulence intensities, particularly in the spanwise direction for this coarse grid resolution. Furthermore, the much coarser grid resolutions of the C395D and C395E cases tested in this study are generally thought to never be applicable to LES. Also, for the higher-Re cases (C1E3F and $\mathrm{C} 5 \mathrm{E} 3 \mathrm{G})$, their grid resolutions were set to be insufficient for LES. In fact, they are much coarser than those used for the LESs in our previous studies[20][21]. Therefore, if the present HLR model provides reasonable predictions for such higher-Re cases, it may be useful from an engineering standpoint.

Next, to illustrate the applicability of the present HLR model for more complex turbulence, we performed calculations for a periodic-hill flow; a schematic view and the grid systems used are shown in Fig. 5. The presence of massive separation between consecutive hills allows the model to be investigated under conditions representative of complex separated flows. For this geometry, highly-resolved LES data were reported by Temmerman et al.[28]. The Reynolds number was 10595, based on the hill height $H$ (channel height: $3.035 \mathrm{H}$ ) and the bulk-mean velocity $\left(U_{b}\right)$ above the hill crest. Three grid resolutions were selected; see summary in Table 2. Note that the spanwise $(z)$ domain was fixed at $4.5 \mathrm{H}$. The grid resolution was controlled by changing the grid numbers, resulting in grid resolutions in the $z$-direction being $\Delta z=0.075 \mathrm{H}, 0.15 \mathrm{H}$ and $0.225 \mathrm{H}$, respectively. For H2DC in particular, the grid resolution is generally too coarse for LES. 
Table 2 Computational parameters for the periodic hill-flow simulations.

\begin{tabular}{lccccc}
\hline \hline Case & Grid nodes & $\Delta x / H$ & $\Delta y / H$ & $\Delta z / H$ & $\Delta t$ \\
\hline H2DF & $161 \times 101 \times 61$ & $0.03-0.082$ & $0.002-0.077$ & 0.075 & $5 \times 10^{-4}$ \\
H2DM & $121 \times 81 \times 31$ & $0.035-0.116$ & $0.002-0.102$ & 0.15 & $8 \times 10^{-4}$ \\
H2DC & $81 \times 61 \times 21$ & $0.04-0.194$ & $0.002-0.145$ & 0.225 & $1 \times 10^{-3}$ \\
\hline \hline
\end{tabular}

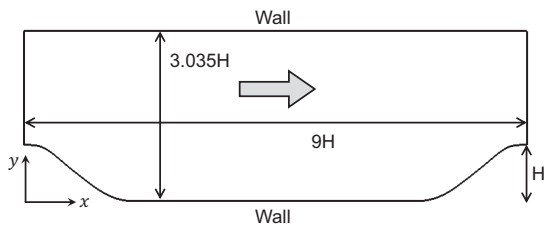

(a) Schematic view

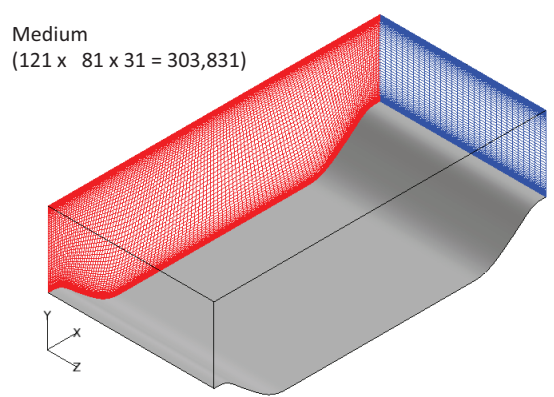

(c) H2DM

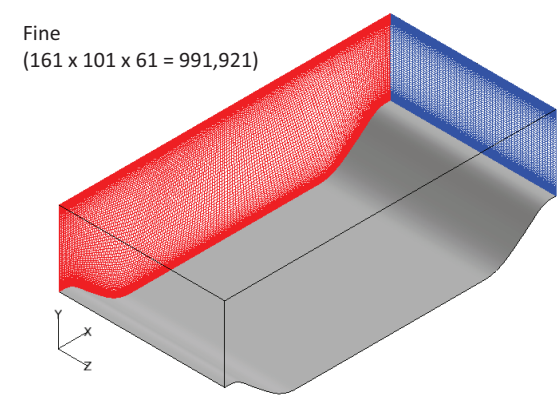

(b) $\mathrm{H} 2 \mathrm{DF}$

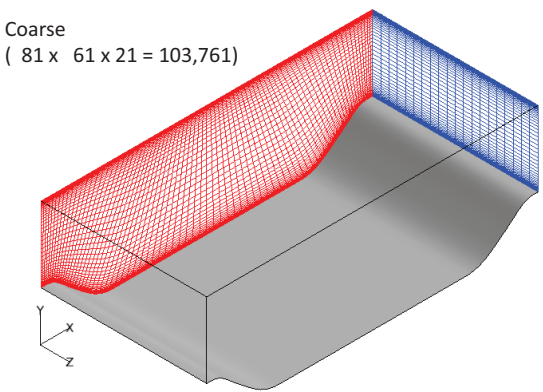

(d) $\mathrm{H} 2 \mathrm{DC}$

Fig. 5 Schematic view and grid system for periodic hill flow.

We performed calculations using an unstructured finite-volume procedure nearly identical to that of Muto et al.[29], where vertex-centered type storage was used on a grid. The second-order central difference scheme was used to discretize the spatial derivatives except for the convection term of the turbulence energy, which was discretized by the second-order upwind scheme. The time marching was based on the fractional step method[30], in which the second-order Crank-Nicolson scheme was used for the velocity equations. On the other hand, the first-order Euler implicit scheme was used for the transport equation of the turbulence energy. The coupling of the velocity and pressure fields was based on the simplified marker and cell method[31]. The flow rate on the control-volume surface was estimated using the Rhie-Chow interpolation[32]. For the boundary conditions, the periodic condition was imposed in the streamwise and spanwise directions, while the no-slip conditions were specified at the wall surfaces. Note that the effects of a first-order time- 
Table 3 Comparison of the bulk-mean velocity $U_{b}$ and the friction coefficient $C_{f}$ with the DNS data[24].

\begin{tabular}{lcccc}
\hline \hline Case & $U_{b}$ & Error (\%) & $C_{f}$ & Error (\%) \\
\hline DNS[24] & 17.55 & & $6.50 \times 10^{-3}$ & \\
C395A & 17.21 & -1.93 & $6.76 \times 10^{-3}$ & 3.98 \\
C395B & 16.97 & -3.29 & $6.95 \times 10^{-3}$ & 6.91 \\
C395C & 18.02 & 2.71 & $6.16 \times 10^{-3}$ & -5.21 \\
C395D & 18.20 & 3.72 & $6.04 \times 10^{-3}$ & -7.05 \\
C395E & 17.98 & 2.48 & $6.19 \times 10^{-3}$ & -4.78 \\
\hline \hline
\end{tabular}

marching scheme and a second-order upwind scheme adopted for the transport equation of turbulence energy were carefully investigated in our previous studies[20][21]. These investigations confirmed that the time-integration and space-discretization schemes used here did not have any crucial effect on the computational results.

\section{Results and Discussion}

\subsection{Basic performance of the present HLR model}

First, to confirm the basic performance of the present HLR model, Fig. 6 compares the distributions of mean velocity, total (resolved+modeled) Reynoldsshear stress, and total turbulence energy at $R e_{\tau}=395$ for various gridresolution cases. The predicted profiles of the mean velocity are smooth and correspond well to the DNS data, although some dispersion is still observed in the distributions. We note that the mean velocity is well predicted even for the coarsest grid-resolution case (C395E). Reasonable prediction of the mean velocity also accounts for the reasonable prediction of the total Reynolds-shear stress. The predicted distributions for the turbulence energy generally agree well with those of the DNS data, although the present HLR model tends to return overpredicted results for coarser grid resolutions.

For a more detailed discussion, the error in the prediction of the skin friction coefficient $C_{f}$ is investigated. Following Piomelli et al.[11], the error is defined as

$$
\text { Error }(\%)=\frac{C_{f}-C_{f, D N S}}{C_{f, D N S}} \times 100, \quad C_{f}=\frac{\tau_{w}}{\rho U_{b}^{2} / 2},
$$

where $\tau_{w}$ and $U_{b}$ denote the mean wall stress and the bulk-mean velocity, respectively. The predicted $C_{f}$ is compared with the DNS data in Fig. 7 and Table 3. Although some dispersion is seen in the $C_{f}$ predictions, the error is confined to within $\pm 7 \%$ and hence the full dispersion in the $C_{f}$ predictions for all grid resolutions has error of about 14\%. From an engineering viewpoint, this level is thought to be acceptable, although the dispersion is expected to be further improved. 


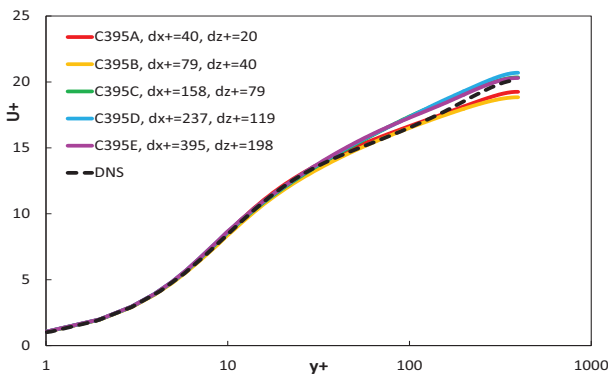

(a) Mean velocity

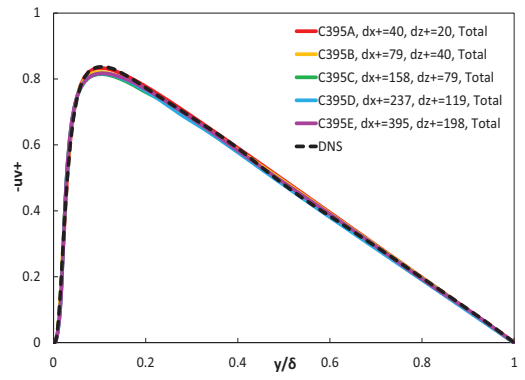

(b) Total $R e$-shear stress

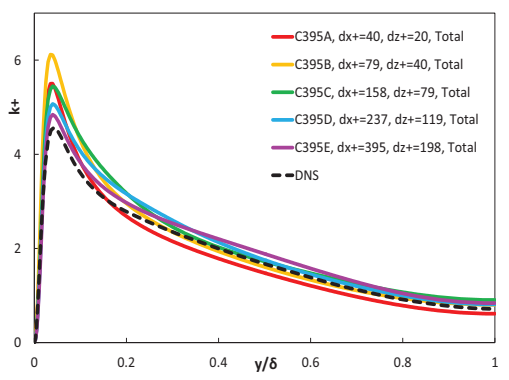

(c) Total turbulence energy

Fig. 6 Comparison of the results for cases at $R e_{\tau}=395$.

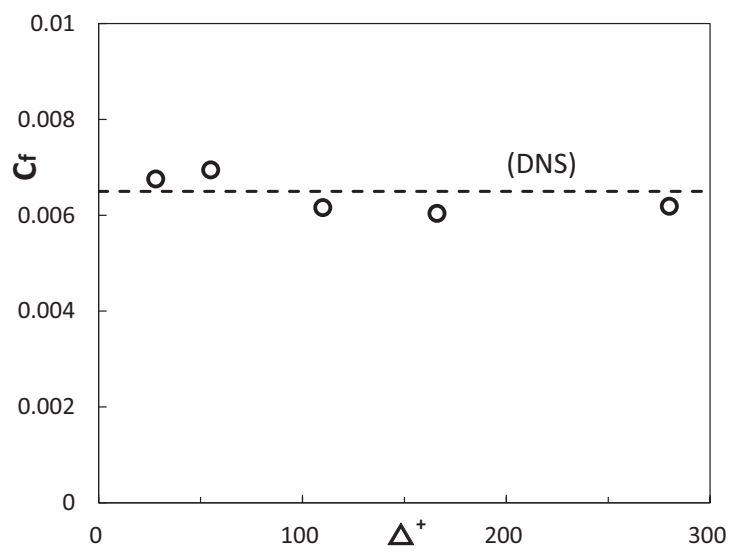

Fig. 7 Comparison of the friction coefficient $C_{f}$ with DNS data[24].

Figure 8 shows the distributions of the total turbulence intensities. Note that the values in the figure were obtained from the root-mean square of the total Reynolds normal stresses. All of the components are generally well predicted, although a close inspection of the results reveals that some scatter remains in the distributions. In particular, the wall-normal intensity for C395E was found to be overpredicted. This result was caused by the extreme grid 


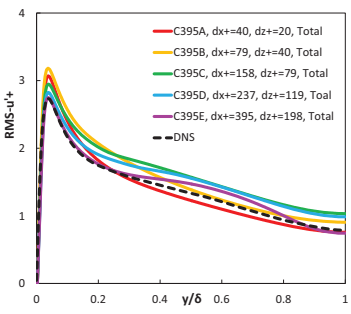

(a) Streamwise

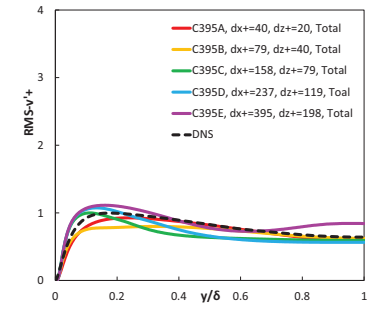

(b) Wall-normal

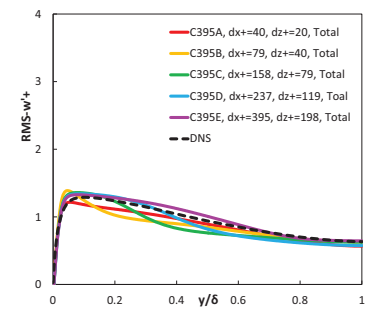

(c) Spanwise

Fig. 8 Comparison of the distributions of total (resolved+modeled) turbulence intensities.

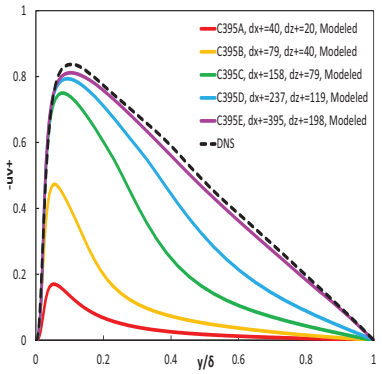

(a) Reynolds shear stress

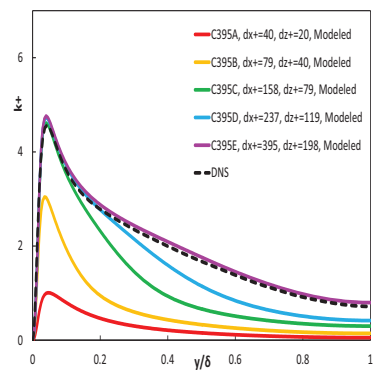

(b) Turbulence energy

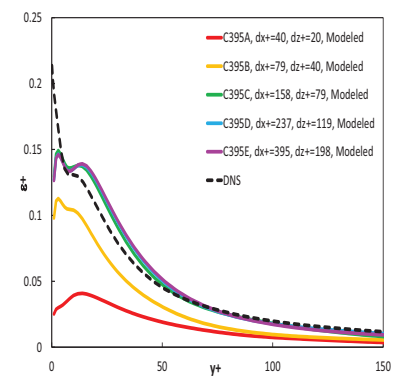

(c) Dissipation rate

Fig. 9 Comparison of the distributions of the modeled values for $R e_{\tau}=395$.

spacing used in this test case. As seen in Table 1, the aspect ratio of the grid spacing in the region far from the wall for C395E is quite large. Because of this large aspect ratio for the grid spacing, the energy redistribution from the streamwise direction to the spanwise direction is considered to be suppressed, resulting in an increase in the wall-normal turbulence compared with that in the spanwise direction.

Figure 9 compares the modeled values of Reynolds-shear stress, turbulence energy, and dissipation rate at $R e_{\tau}=395$ for various grid resolutions. A reasonable trend is obtained that the modeled value increases as the grid resolution becomes coarser. Particularly, for the coarsest grid-resolution case (C395E), the computational results of the modeled values resemble those of the DNS statistics because RANS becomes more dominant. A close inspection however reveals that some discrepancy is still seen in the prediction of $\varepsilon$ even for this coarsest grid-resolution case. In particular, the peak value of $\varepsilon$ at the wall surface is not reproduced. This fact is thought to be closely related to the slight underprediction of $k$ in the vicinity of the wall. Such a trend has often been seen in results predicted by a number of low-Re RANS models. However, this issue may be improved in the future once the model constant $\sigma_{k}$ is made spatially variable to enhance the turbulent diffusion in the near-wall region (see, for example, Abe[23]; Abe et al.[33]). 


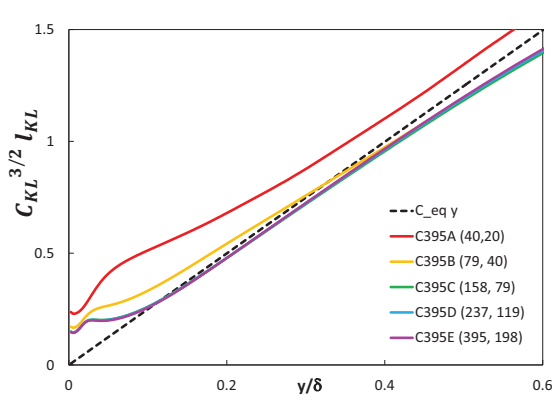

(a) Comparison with $C_{e q} y$

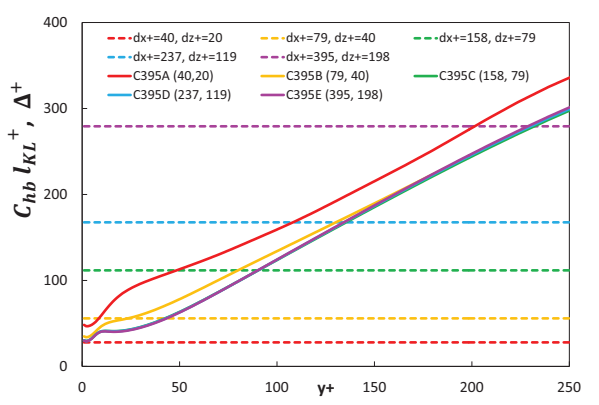

(b) Comparison with $\Delta^{+}$

Fig. 10 Comparison of the distributions of switching length-scale parameter.

4.2 Detailed discussion of the present length-scale parameter

Figure 10 shows the distributions of the length-scale parameter defined by Eq. (22) at $R e_{\tau}=395$ for various grid-resolution cases. Note that the values of $l_{K L}^{+}$in Fig. 10 (b) are multiplied by $C_{h b}$ for comparison with the grid resolution $\Delta^{+}$in Table 1 (see, Eq. (27) for detail).

We find from Fig. 10 (a) that the distributions of $l_{K L}$ are basically similar to those of the a priori tests shown in Fig. 3 (a), although discrepancies can be observed in the finer grid-resolution cases (C395A and C395B). These discrepancies correspond to Fig. 9 (c), where the dissipation rate in the LES region tends to decrease as the grid resolution becomes finer. Although this result is a natural consequence of the basic concept of the present HLR model, we need to discuss carefully whether it may result in any crucial problems in real calculations.

A detailed investigation of the length-scale distributions in Fig. 10 (b) provides us with several important findings. Considering the expression of the present switching function in Eq. (27), the switching between LES and RANS roughly occurs at the location of $C_{h b} l_{K L}^{+} \sim \Delta^{+}$. This location is indicated in Fig. 10 (b) as the point at which two lines of the same color cross each other. As already described in Fig. 10 (a), the red line of $l_{K L}^{+}$for C395A considerably deviates from the other distributions (i.e., the green, blue and purple lines). This deviation is caused by the small value of the dissipation rate in the LES region. Although this value is much smaller than that originally evaluated by the RANS statistics in an a priori test, its effect generally tends to increase the estimated length scale. As a result, the two red lines do not cross each other in Fig. 10 (b). This fact means that the full LES region is successfully maintained in the case that the grid resolution is sufficiently fine. Therefore, this a posteriori test indicates that the present length-scale model sufficiently works even in the full LES condition, where the length scale must be evaluated by the SGS dissipation rate in real calculations, although further improvement may be possible by taking this fact into account. 


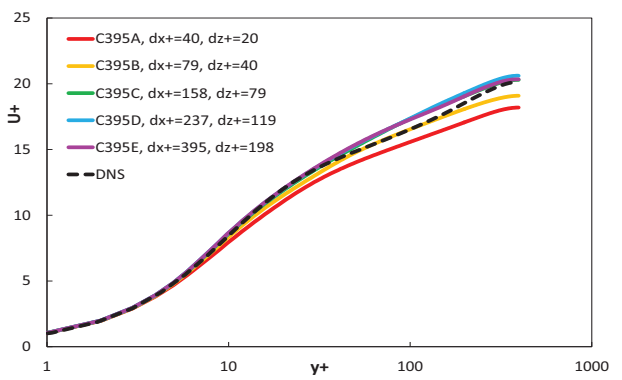

Fig. 11 Computational results of the mean velocity using the conventional switching function Eq. (29) for $R e_{\tau}=395$.

Next, to discuss the effect of the switching function on the model performance, we investigated the results of additional computations using the conventional switching function (Eq. (29)) for tests with $R e_{\tau}=395$. The predicted mean-velocity distributions are shown in Fig. 11. As readily found in the figure, the mean velocity for C395A is considerably underpredited, although the other predictions are similar to those of the present HLR model. Indeed, the calculated friction coefficient for C395A is $C_{f}=7.66 \times 10^{-3}$ and thus the error defined by Eq. (30) reaches about 18\%, which is much larger than that of the present HLR model.

Figure 12 shows the distributions of the switching function $f_{h b}$ at $R e_{\tau}=$ 395 for various grid-resolution cases. In the figure, the distributions for $f_{h b}$ obtained using both the present (Eq. (27)) and the conventional (Eq. (29)) functions are shown. As already described in Fig. 10 (b), the switching location between LES and RANS for the present HLR model is roughly located where two lines of the same color cross each other, i.e., $C_{h b} l_{K L}^{+} \sim \Delta^{+}$. In this sense, the profiles of $f_{h b}$ in Fig. 12 (a) generally account for those of $l_{K L}^{+}$in Fig. 10 (b). Furthermore, we confirm that an almost full LES is successfully achieved for the finest grid-resolution case (C395A). This trend is almost the same as that expected in an a priori test (see, Fig. 4).

In contrast, $f_{h b}$ in Fig. 12 (b) shows a different aspect that the conventional switching function goes to zero in the near-wall region even for C395A. As seen in the figure, $f_{h b}$ is roughly 0.5 at around $x / \delta \sim 0.05$ which implies $y^{+} \sim 20$ for this Reynolds number $\left(R e_{\tau}=395\right)$. As the buffer layer is involved in the switching region, the model performance is considered to be largely affected. This is the main reason why the prediction accuracy of the conventional model was worse than that of the present model particularly for C395A (Fig. 11).

\subsection{Higher Reynolds-number cases}

To assess the predictive performance of the present HLR model in more detail, we investigate the computational results for higher-Re cases. Figure 13 compares the predictions of mean velocity, Reynolds-shear stress and turbu- 


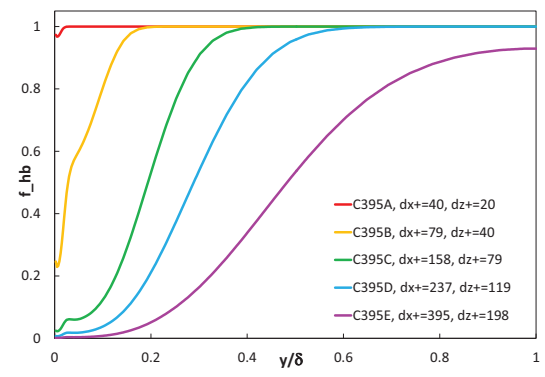

(a) The present model

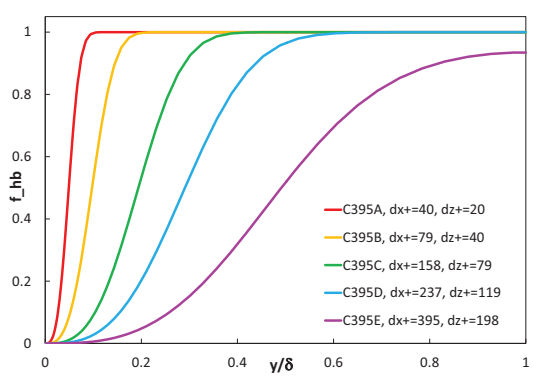

(b) Conventional model

Fig. 12 Comparison of the distributions of the switching function $f_{h b}$.

lence energy for C1E3F with the corresponding DNS data[25]. We can see in Fig. 13 (a) that the mean velocity is generally well predicted. Although a slight discrepancy is still observed in the logarithmic region, the error in the prediction of $C_{f}$ defined by Eq. (30) was only about $-1 \%\left(C_{f}=4.80 \times 10^{-3}\right.$, $C_{f, D N S}=4.85 \times 10^{-3}$ ) and thus the prediction accuracy is considered to be acceptable. As seen in Fig. 13 (b), the computational results of the total Reynolds shear stress correspond well to the DNS data. The total turbulence energy compared in Fig. 13 (c) is generally well predicted. Although some discrepancies are still seen in the predictions, the present results given for such a coarse grid resolution (i.e., $\Delta x^{+}=250, \Delta z^{+}=125$, see Table 1) are thought to be encouraging.

Figure 14 shows the computational results of mean velocity and total and modeled Reynolds-shear stresses for various $R e$ cases, including the highest Re case (C5E3G). From Fig. 14 (a) and (b), the predicted distributions of the mean velocity and the total Reynolds-shear stress generally correspond to wellknown studies of this type of wall-shear flows. A closer inspection reveals that an upward shift occurs in the logarithmic region compared with the generally used log-law line, i.e., $\bar{U}^{+}=(1 / 0.41) \log y^{+}+5.0$. These predictions lie close to an alternative known correlation, i.e., $\bar{U}^{+}=(1 / 0.4) \log y^{+}+5.5$.

To clarify the upward shift in the predicted mean velocities, the results of a one-dimensional full RANS calculation preliminary performed are shown in Fig. 15 for reference. Because the computational cost for this calculation was negligible, the Reynolds number was increased to $R e_{\tau}=1.3 \times 10^{6}$ (i.e., the bulk Reynolds number $\left.R e_{b}=2 U_{b} \delta / \nu \sim 10^{8}\right)$. As seen in the figure, the log-law line is maintained even for very high-Re values and the profiles of the Reynolds-shear stress do not contradict current understanding of this flow. However, an upward shift similar to Fig. 14 (a) is also seen in Fig. 15 (a). As characteristics of the RANS model are expected to appear in the nearwall region for coarser grid resolutions, the upward shift in Fig. 14 (a) is considered to derive mainly from the performance of the one-equation RANS model adopted. In this regard, this upward shift is different from that caused by the "double-buffer problem." 


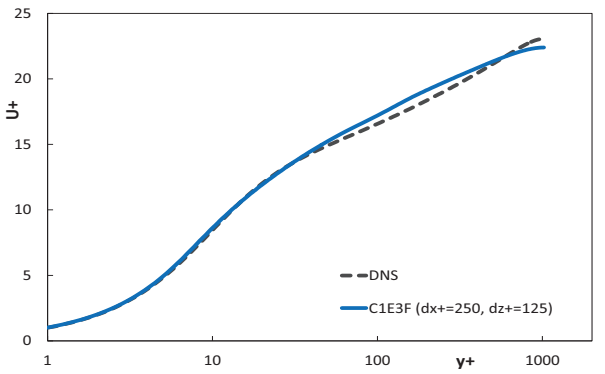

(a) Mean velocity

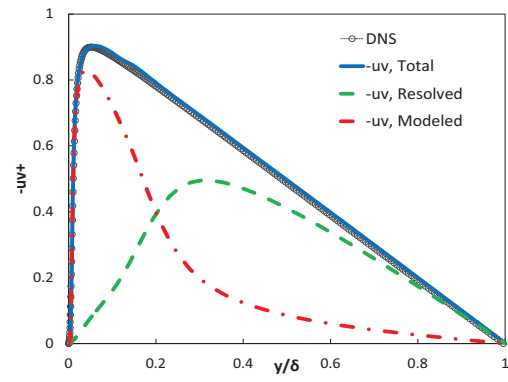

(b) Reynolds shear stress

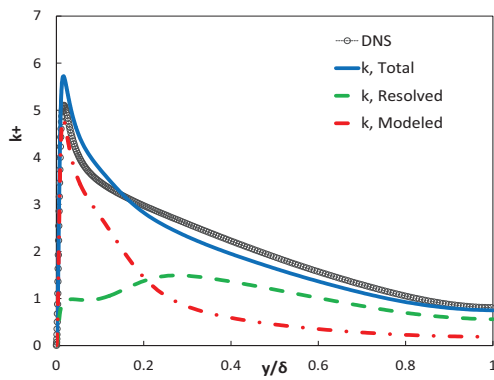

(c) Turbulence energy

Fig. 13 Comparison of the results with the DNS data[25] for C1E3F.

Nevertheless, we recognize from Fig. 14 (c) that, for C5E3G in particular, the region covered by RANS was only about one tenth (or less) of the entire domain, the remaining region was covered by LES. As noted earlier, this is achieved by adopting an advanced anisotropy-resolving SGS model in the LES region[20][21]. Considering the coarse grid resolution used in C5E3G (i.e., $\Delta x^{+}=333, \Delta z^{+}=167$ ), the computational results are encouraging from an engineering perspective, although the aforementioned upward shift in the logarithmic region needs to be improved.

\subsection{Periodic hill-flow}

To investigate the model performance for more complex turbulence with flow separation, the computational results from hill-flow simulations are compared with the corresponding highly-resolved LES data of Temmerman et al. (2003) (hereafter "reference data"). Figure 16 compares the model-predicted streamlines. As readily seen in the figure, the separation bubble is predicted generally well with the present HLR model at all grid resolutions. It is also understood from Fig. 16 (b) that an almost full LES is achieved for the finest grid-resolution case (H2DF). Moreover, we see in Fig. 16 (d) that the distribution of $f_{h b}$ looks reasonable and shows no unexpected behavior even in the separation bubble for the coarsest grid-resolution case (H2DC). 


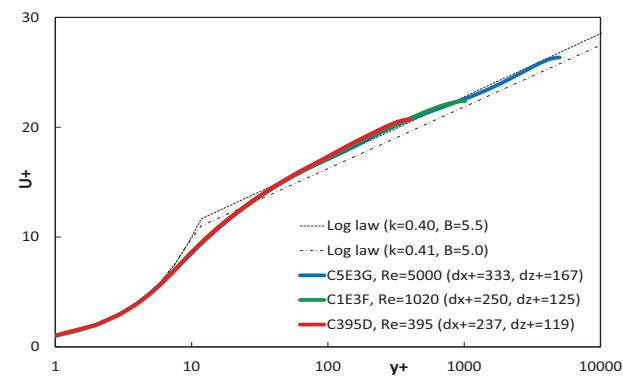

(a) Mean velocity

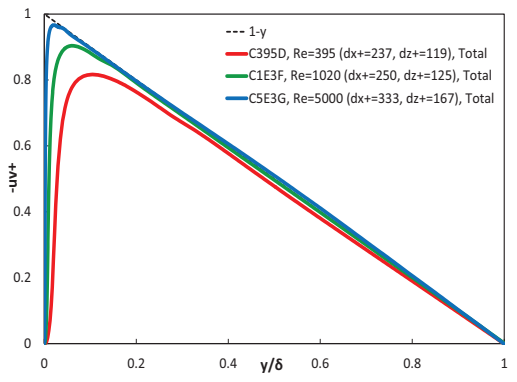

(b) Total $R e$-shear stress

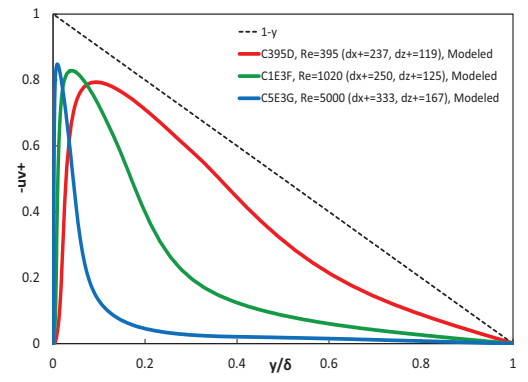

(c) Modeled $R e$-shear stress

Fig. 14 Computational results for various $R e$ values.

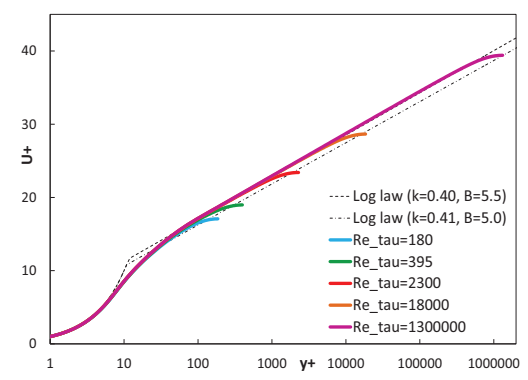

(a) Mean velocity

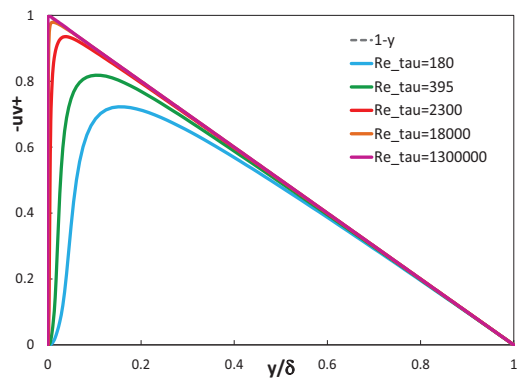

(b) Re-shear stress

Fig. 15 Computational results of the one-dimensional full-RANS calculation for reference.

The predicted positions of the separation $\left(X_{S} / H\right)$ and the reattachment $\left(X_{R} / H\right)$ are compared with the reference data in Table 4 . Note that the other two highly-resolved LESs (Breuer et al.[18] and Froehlich et al.[34]) are also reliable and thus the separation and reattachment points obtained by these LESs are included for greater comparison. Furthermore, Fig. 17 compares the length and location of the separation bubbles illustrated using these data. The predictions are in good agreement with the reference data and their grid dependency is sufficiently small. Comparison of the predictions for the friction coefficient $C_{f}$ and the pressure coefficient $C_{P}$ on the lower wall is given in 


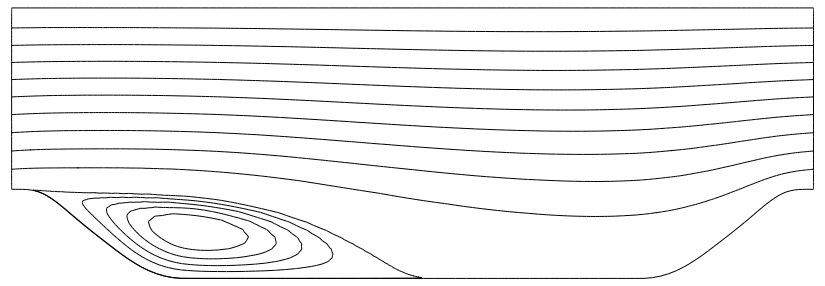

(a) Reference (Temmerman et al.[28])

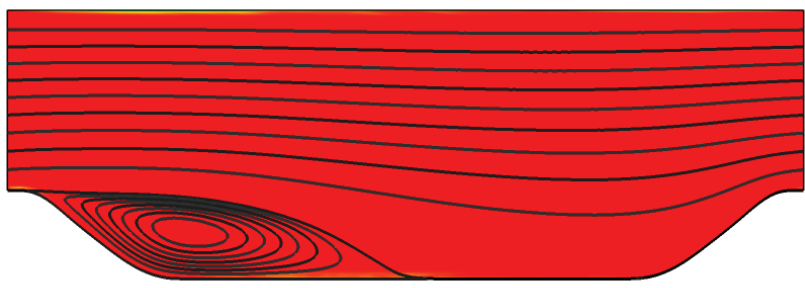

(b) $\operatorname{H} 2 \mathrm{DF}(161 \times 101 \times 61)$

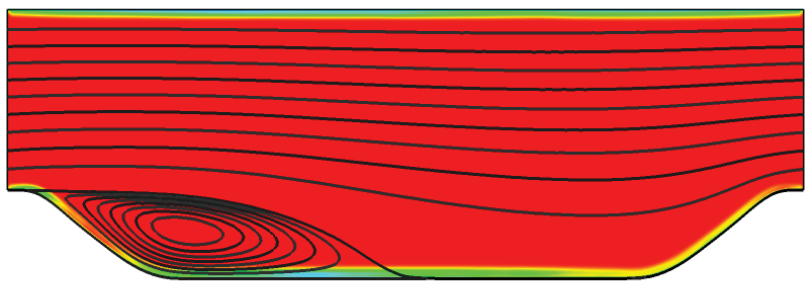

(c) $\mathrm{H} 2 \mathrm{DM}(121 \times 81 \times 31)$

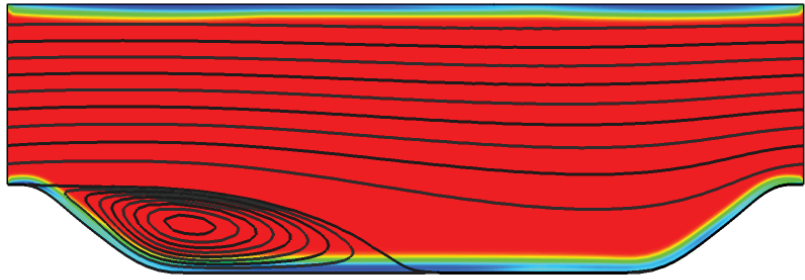

(d) $\operatorname{H} 2 \mathrm{DC}(81 \times 61 \times 21)$

Fig. 16 Comparison of streamlines for the periodic hill flow (color shows the switching function $f_{h b}$, which ranges from 0 (Blue) to 1 (Red)).

Fig. 18. With very slight dispersion, these distributions show good agreement with the reference data.

Figure 19 compares the computational results of the mean velocity and the total (GS+SGS) Reynolds-shear stress at five streamwise positions, i.e., $x / H=0.5,2,4,6$ and 8 . The figures show that the predictions are generally in good agreement with the reference data for all grid resolutions, although 
Table 4 Comparison of the separation $\left(X_{S} / H\right)$ and reattachment $\left(X_{R} / H\right)$ points for the periodic hill flow.

\begin{tabular}{lcccccc}
\hline \hline Case & Ref.[18] & Ref.[28][34] & Ref.[34] & H2DF & H2DM & H2DC \\
\hline$X_{S} / H$ & 0.19 & 0.22 & 0.20 & 0.19 & 0.20 & 0.20 \\
$X_{R} / H$ & 4.69 & 4.72 & 4.56 & 4.81 & 4.81 & 4.91 \\
\hline \hline
\end{tabular}

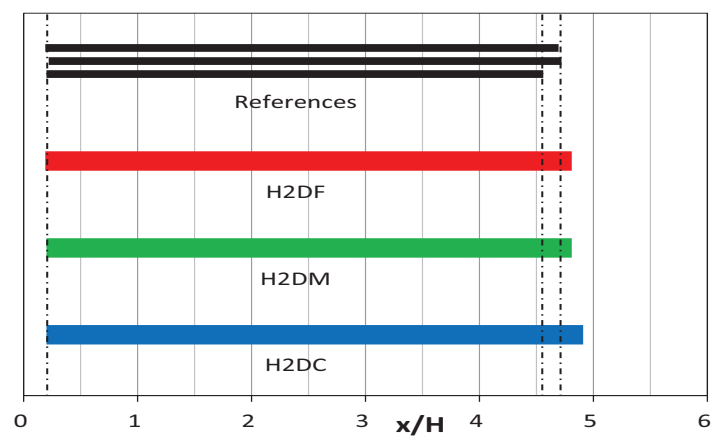

Fig. 17 Comparison of length and location of the separation bubble predicted in the periodic hill-flow case.

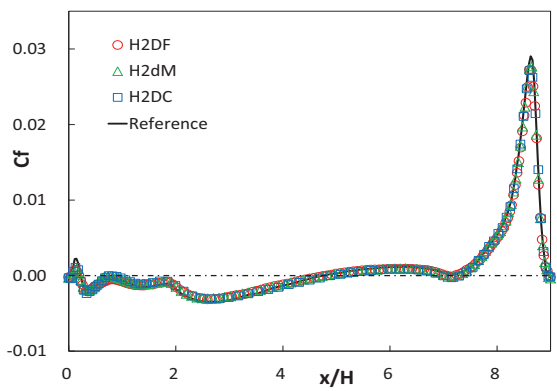

(a) Friction coefficient $C_{f}$

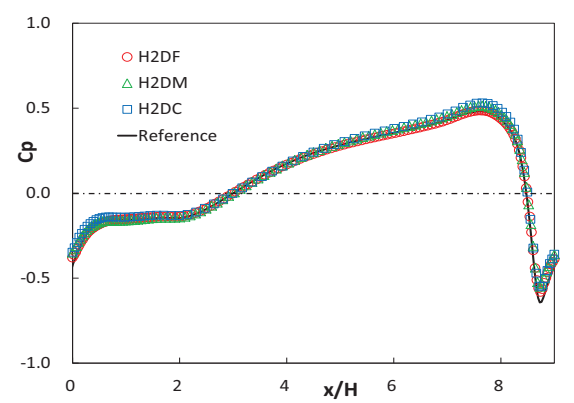

(b) Pressure coefficient $C_{P}$

Fig. 18 Comparison of the friction coefficient $C_{f}$ and the pressure coefficient $C_{P}$ on the lower wall for the periodic hill flow.

we still see slight discrepancies particularly in the predictions of the Reynoldsshear stress. Overall, these results indicate the basic applicability of the present HLR model to complex turbulence with flow separation.

Finally, note that the number of grid nodes in $x-y$ plane used for the finest grid-resolution case $(\mathrm{H} 2 \mathrm{DF})$ is indeed the same as that for the HLR simulations in Breuer et al.[18]. As already found in Fig. 16 (b), however, the present model provides almost full LES results for this grid resolution with sufficient prediction accuracy. Furthermore, the computational results infer that even if the number of grid nodes is reduced to about one-tenth of H2DF (i.e., H2DC), the present HLR model still works properly, at least for this 

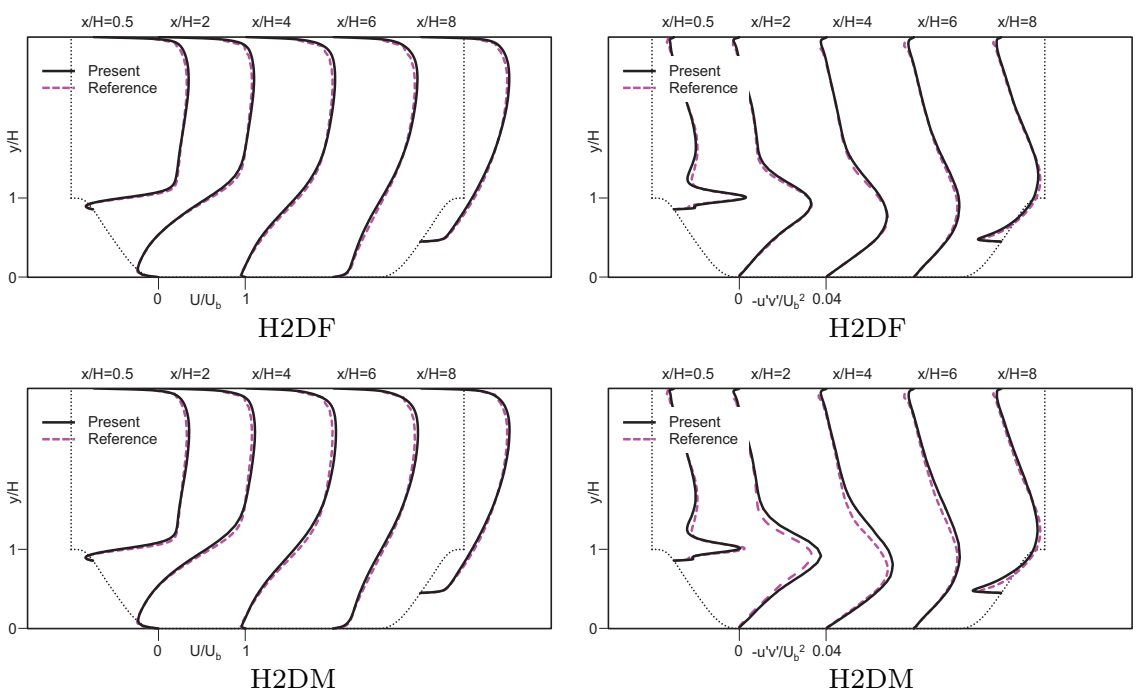

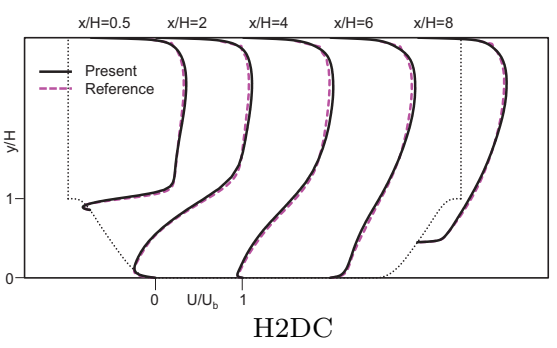

(a) Mean velocity

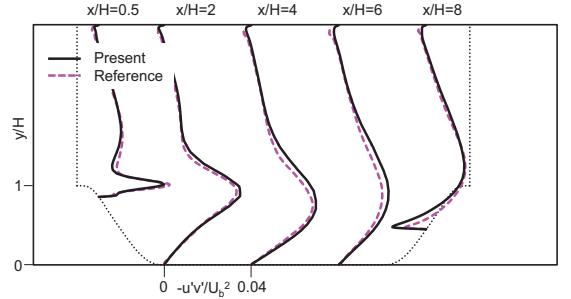

$\mathrm{H} 2 \mathrm{DC}$

(b) Total Re-shear stress

Fig. 19 Computational results of the mean velocity and the total (GS+SGS) Reynoldsshear stress for the periodic hill flow.

hill-flow case. This fact is very encouraging and prompts further development of this research field.

\section{Concluding Remarks}

An effective switching parameter was newly proposed for an advanced HLR model. This switching parameter introduced knowledge of the Kolmogorov microscale that is thought to be reasonable for representing near-wall turbulence. In contrast with most previous models, the present HLR model is smoothly replaced by a full LES if a grid resolution is fine enough in the near-wall region. Furthermore, the present HLR model adopted an anisotropy-resolving SGS model in the LES region. By using this SGS model, the present HLR model can successfully cover a much wider range of flow domain by LES compared with the model previously proposed by our group. To connect the LES and RANS regions smoothly, we also developed a one-equation NLEVM as the RANS model that was successfully connected with the aforementioned 
one-equation SGS model. To assess the basic performance of the model, the proposed HLR model was applied to numerical simulations of a periodic hill flow as well as fundamental plane channel flows.

For the low- $R e$ channel-flow cases at $R e_{\tau}=395$, the predicted profiles of mean velocity, Reynolds-shear stress, and turbulence energy generally corresponded well to the DNS data for all of the tested grid resolutions. All components of the turbulence intensities were also generally well predicted. Concerning the balance between resolved and modeled components, the modeled component increased as expected when the grid resolution becomes coarser. For higher-Re cases, the present HLR model generally returned reasonable predictions for the mean velocity with a grid resolution too coarse for pure LES, although some upward shift was still seen in the logarithmic region.

In regard to the periodic hill-flow case, the predictions using the present HLR model were very encouraging. For the finest grid-resolution case, almost full LES was achieved and its predictions were in good agreement with the reference data. In contrast, the RANS properly covered the near-wall region for the coarsest grid-resolution case, where the predicted size of the separation bubble still showed good agreement with the reference data. In all tests, the mean velocity and the Reynolds-shear stress were well predicted with only a small grid dependency. Therefore, the model performance is considered to be generally acceptable for the tests done.

A detailed investigation of the length-scale model and the switching function proposed in this study elucidated that the new idea was effective for developing an advanced HLR model. An a posteriori test confirmed that the present length-scale model worked well, even in full LES conditions, where it must be evaluated by the SGS dissipation rate in real calculations. Although the dissipation rate in this condition is much smaller than that originally evaluated by the RANS statistics in an a priori test, its effect generally tends to make the evaluated length scale increase, while still maintaining the full LES condition. Therefore, this discrepancy does not cause any crucial problem in the prediction. The application to a periodic hill flow also showed that the present switching function worked appropriately even in a separated region. These findings indicate a basic prospect of the present HLR model from an engineering viewpoint.

Finally, we briefly note the following as areas of future study. The Kolmogorov microscale based on the dissipation rate was introduced in the switching parameter as a means to represent turbulent structures. However, the near-wall dissipation rate brings about a strong anisotropic feature. From this aspect, the use of the near-wall Kolmogorov scale needs further discussion. Nevertheless, many studies reported that, instead of the friction velocity, the Kolmogorov scale works well as a scaling parameter of turbulence. Although the present idea worked even in the application to a periodic hill flow, more detailed investigations will contribute to further development.

Although the predicted results were generally acceptable, the mean-velocity predictions for channel-flow cases revealed an upward shift in the logarithmic region compared with the well-known log-law line. This derived from the per- 
formance of the RANS model adopted in this study. The present algebraic $\epsilon$ model was simply constructed by a priori tests using the DNS data. Therefore, further adjustments may be necessary through a posteriori tests, although several factors must be taken into account before re-optimization.

On the other hand, the so-called "gray zone" where $f_{h b} \sim 0.5$ may be another important issue to be investigated. Actually, it is not easy to give a completely physics-oriented explanation for Eq. (1). Moreover, it is still unclear whether a zonal or a non-zonal approach is better for constructing a further advanced HLR model. Although the present HLR model regarded as a zonal approach worked generally well with no "double-buffer problem" for all grid resolutions tested, this issue needs further discussion.

In this study, we dealt mainly with wall-shear turbulent flows because the smallest scale of turbulent structures are generally seen in the near-wall region. On the other hand, at this stage, the present switching function is constructed to choose LES for a far-field free-shear turbulent region because it seems better to use LES rather than RANS for such an external flow even if the three-dimensional grid resolution is a little insufficient for LES. Therefore, it is left to future studies how to introduce RANS effectively in a far field. Although this issue will be solved by the progress of a high-performance super computer before the development of a sophisticated RANS model, the problem of the aforementioned near-wall turbulence at high $R e$ will remain to the end of the discussion in this research field. In this sense, the present HLR model still stands on the first step of the development, although the basic performance of the model is considered to be very encouraging.

Acknowledgements This research was partially supported by Grant-in-Aid for Scientific Research 24560197, sponsored by the Japan Society for the Promotion of Science. This research was also supported by the "Advanced Computational Scientific Program" of the Research Institute for Information Technology, Kyushu University, Japan. The present computation was primarily carried out using the computer facilities at the Research Institute for Information Technology, Kyushu University, Japan.

\section{Appendix}

In this study, the modeling concept proposed by Abe et al.[23] was introduced to develop a one-equation NLEVM used in the RANS region. In the following section, we briefly describe the model expressions followed by several minor modifications made by Abe[16]. The Reynolds stress $\overline{u_{i} u_{j}}$ is modeled as follows:

$$
\begin{aligned}
& \overline{u_{i} u_{j}}=\frac{2}{3} k \delta_{i j}-2 k \tau C_{B}\left[1+\left\{1-f_{w}(26)\right\} f_{s 1}\right] S_{i j} \\
& +4 k \tau^{2} C_{D} C_{B}\left\{1-f_{w}(26)\right\}\left\{-\left(S_{i k} \Omega_{k j}-\Omega_{i k} S_{k j}\right)\right. \\
& \left.+\left(1-f_{s 2}\right)\left(S_{i k} S_{k j}-\frac{\delta_{i j}}{3} S^{2}\right)\right\} \\
& +2 k^{w} b_{i j} .
\end{aligned}
$$


In Eq. (31), the model function $f_{w}$ is modeled as follows[33]:

$$
f_{w}(\xi)=\exp \left\{-\left(\frac{n^{*}}{\xi}\right)^{2}\right\}
$$

where $\xi$ is a prescribed constant. In Eq. $(32), n^{*}\left(=(\nu \varepsilon)^{1 / 4} n / \nu\right)$ is the nondimensional wall distance with the Kolmogorov scale[27], where $n$ is uniquely determined as the shortest distance from all of the wall surfaces. In the model, the characteristic time scale $\tau$ and the model coefficients are given as follows[23]:

$$
\begin{gathered}
\tau=\frac{\nu_{t}}{k}, \quad \nu_{t}=C_{\mu} f_{\mu} \frac{k^{2}}{\varepsilon}, \quad f_{\mu}=\left[1+\frac{35}{R_{t}^{\frac{3}{4}}} \exp \left\{-\left(\frac{R_{t}}{30}\right)^{\frac{3}{4}}\right\}\right]\left\{1-f_{w}(26)\right\}, \\
C_{B}=\frac{1}{1+\frac{22}{3}\left(C_{D} \tau\right)^{2} \Omega^{2}+\frac{2}{3}\left(C_{D} \tau\right)^{2}\left(\Omega^{2}-S^{2}\right) f_{B}}, \\
f_{s 1}=f_{r 1} f_{r 2} C_{s 1}\left(C_{D} \tau\right)^{2}\left(\Omega^{2}-S^{2}\right), \quad f_{s 2}=f_{r 1} f_{r 2}\left\{1+C_{s 2} C_{D} \tau(\Omega-S)\right\} \\
f_{B}=1+C_{\eta} C_{D} \tau(\Omega-S), \quad f_{r 1}=\frac{\Omega^{2}-S^{2}}{\Omega^{2}+S^{2}}, \quad f_{r 2}=\frac{S^{2}}{\Omega^{2}+S^{2}}, \\
S^{2}=S_{m n} S_{m n}, \quad \Omega^{2}=\Omega_{m n} \Omega_{m n}, \quad S=\sqrt{S^{2}}, \quad \Omega=\sqrt{\Omega^{2}}, \\
C_{D}=0.8, \quad C_{\mu}=0.12, \quad C_{\eta}=100, \quad C_{s 1}=0.15 C_{\eta}, \quad C_{s 2}=0.07 C_{\eta},
\end{gathered}
$$

where $R_{t}\left(=k^{2} / \nu \varepsilon\right)$ is the turbulent Reynolds number.

In Eq. (31), ${ }^{w} b_{i j}$ is introduced to improve the predictive performance of the near-wall stress anisotropy as follows[23]:

$$
\begin{aligned}
{ }^{w} b_{i j}=f_{w}(26)\left[-\alpha_{w} \frac{1}{2}\right. & \left(d_{i} d_{j}-\frac{\delta_{i j}}{3} d_{k} d_{k}\right) \\
+\left(1-f_{r 1}{ }^{2}\right) \tau_{d}^{2}\{ & -\frac{\beta_{w} C_{w}}{1+C_{w} \tau_{d}^{2} \sqrt{S^{2} \Omega^{2}}}\left(S_{i k} \Omega_{k j}-\Omega_{i k} S_{k j}\right) \\
& \left.\left.+\frac{\gamma_{w} C_{w}}{1+C_{w} \tau_{d}^{2} S^{2}}\left(S_{i k} S_{k j}-\frac{\delta_{i j}}{3} S^{2}\right)\right\}\right]
\end{aligned}
$$

where

$$
d_{i}=\frac{N_{i}}{\sqrt{N_{k} N_{k}}}, \quad N_{i}=\frac{\partial n}{\partial x_{i}}, \quad \tau_{d}=\left\{1-f_{w}(15)\right\} \frac{k}{\varepsilon}+f_{w}(15) \delta_{w} \sqrt{\frac{\nu}{\varepsilon}} .
$$

In this study, the following combined model is used to represent the near-wall fragment[16]:

$$
\begin{gathered}
{ }^{w} b_{i j}=f_{\tau}{ }^{w 1} b_{i j}+\left(1-f_{\tau}\right){ }^{w 2} b_{i j}, \quad f_{\tau}=\exp \left[-3\left\{1-f_{w}(26)\right\} \frac{k}{\varepsilon} \sqrt{S^{2}}\right], \\
{ }^{w 1} b_{i j}=\left(\alpha_{w}=1, \quad \beta_{w}=\frac{1}{4}, \quad \gamma_{w}=1.5, \quad \delta_{w}=1.0, \quad C_{w}=0.5\right), \\
{ }^{w 2} b_{i j}=\left(\alpha_{w}=0, \quad \beta_{w}=\frac{13}{30}, \quad \gamma_{w}=0.6, \quad \delta_{w}=3.0, \quad C_{w}=1.0\right) .
\end{gathered}
$$

Further detailed descriptions are given in Abe[16] and Abe et al.[23]. 


\section{References}

1. Smagorinsky, J.: General circulation experiments with the primitive equations. I. The basic experiment. Mon. Weather Rev. 91, 99-164 (1963).

2. Bardina, J., Ferziger, J.H., Reynolds, W.C.: Improved subgrid scale models for large eddy simulation, AIAA Paper, No. 80-1357 (1980).

3. Germano, M., Piomelli, U., Moin, P., Cabot, W.H.: A dynamic subgrid-scale eddy viscosity model, Phys. Fluids A 3, 1760-1765 (1991).

4. Lilly, D.K.: A proposed modification of the Germano subgridscale closure method. Phys. Fluids A 4, 633-635 (1992).

5. Zang, Y., Street, R.L., Koseff, J.R.: A dynamic mixed subgrid-scale model and its application to turbulent recirculating flows, Phys. Fluids A 5, 3186-3196 (1993).

6. Horiuti, K.: A new dynamic two-parameter mixed model for large-eddy simulation, Phys. Fluids 9, 3443-3464 (1997).

7. Morinishi, Y., Vasilyev, O.V.: A recommended modification to the dynamic twoparameter mixed subgrid scale model for large eddy simulation of wall bounded turbulent flow, Phys. Fluids 13, 3400-3410 (2001).

8. Balaras, E., Benocci, C., Piomelli, U.: Two-layer approximate boundary conditions for large-eddy simulations. AIAA J. 34, 1111-1119 (1996).

9. Nikitin, N.V., Nicoud, F., Wasistho, B., Squires, K.D., Spalart, P.R.: An approach to wall modeling in large-eddy simulations. Phys. Fluids 12, 1629-1632 (2000).

10. Hamba, F.: An attempt to combine large eddy simulation with the $k-\varepsilon$ model in a channel-flow calculation. Theoret. Comput. Fluid Dynamics 14, 323-336 (2001).

11. Piomelli, U., Balaras, E., Pasinato, H., Squaires, K.D., Spalart, P.R.: The inner-outer layer interface in large-eddy simulations with wall-layer models. Int. J. Heat and Fluid Flow 24, 538-550 (2003).

12. Davidson, L., Peng, S.H.: Hybrid LES-RANS modelling: a one-equation SGS model combined with a $k-\omega$ model for predicting recirculating flows. Int. J. Numer. Meth. Fluids 43, 1003-1018 (2003).

13. Batten, P., Goldberg, U., Chakravarthy, S.: Interfacing statistical turbulence closures with large-eddy simulation. AIAA J. 42, 485-492 (2004).

14. Hanjalic, K, Hadziabdic, M., Temmerman, L., Leschziner, M.A.: Merging LES and RANS strategies: zonal or seamless coupling? Direct and Large Eddy Simulation V (R. Friedrich et al. (eds)), Kluwer Academic Publ., 451-464 (2004).

15. Temmerman, L., Hadziabdic, M., Leschziner, M.A., Hanjalic, K.: A hybrid two-layer URANS-LES approach for large eddy simulation at high Reynolds numbers. Int. J. Heat and Fluid Flow 26, 173-190 (2005).

16. Abe, K.: A hybrid LES/RANS approach using an anisotropy-resolving algebraic turbulence model, Int. J. Heat Fluid Flow 26, 204-222 (2005).

17. Spalart, P.R., Deck, S., Shur, M.L., Squires, K.D., Strelets, M.Kh., Travin, A.: A new version of detached-eddy simulation, resistant to ambiguous grid densities. Theoret. Comput. Fluid Dynamics 20, 181-195 (2006).

18. Breuer, M., Jaffrezic, B., Arora, K.: Hybrid LES/RANS technique based on a oneequation near-wall model, Theoret. Comput. Fluid Dynamics 22, 157-187 (2008).

19. Deck, S.: Recent improvements in the Zonal Detached Eddy Simulation (ZDES) formulation. Theoret. Comput. Fluid Dynamics 26, 523-550 (2012).

20. Abe, K.: An improved anisotropy-resolving subgrid-scale model with the aid of a scalesimilarity modeling concept, Int. J. Heat Fluid Flow 39, 42-52 (2013).

21. Abe, K.: An investigation of SGS-stress anisotropy modeling in complex turbulent flow fields, Flow, Turbulence and Combustion 92, 503-525 (2014).

22. Inagaki, M.: A new wall-damping function for large eddy simulation employing Kolmogorov velocity scale, Int. J. Heat Fluid Flow 32, 26-40 (2011).

23. Abe, K., Jang, Y. J., Leschziner, M. A.: An investigation of wall-anisotropy expressions and length-scale equations for non-linear eddy-viscosity models, Int. J. Heat Fluid Flow 24, 181-198 (2003).

24. Moser, R.D., Kim, J., Mansour, N. N.: Direct numerical simulation of turbulent channel flow up to $R e_{\tau}=590$, Phys. Fluids 11, 943-945 (1999). 
25. Abe, H., Kawamura, H., Matsuo, Y.: Surface heat-flux fluctuations in a turbulent channel flow up to $R e_{\tau}=1020$ with $\mathrm{Pr}=0.025$ and 0.71 ., Int. J. Heat Fluid Flow 25, 404-419 (2004).

26. Nagano, Y., Tagawa, M.: An improved $k-\varepsilon$ model for boundary layer flows, J. Fluids Engineering 112, 33-39 (1990).

27. Abe, K., Kondoh, T., Nagano, Y.: A New Turbulence Model for Predicting Fluid Flow and Heat Transfer in Separating and Reattaching Flows - I. Flow Field Calculations, Int. J. Heat Mass Transfer 37, 139-151 (1994).

28. Temmerman, L., Leschziner, M.A., Mellen, C.P., Froehlich, J.: Investigation of wallfunction approximations and subgrid-scale models in Large Eddy Simulation of separated flow in a channel with streamwise periodic constrictions. Int. J. Heat and Fluid Flow 24, 157-180 (2003).

29. Muto, M., Tsubokura, M., Oshima, N.: Negative Magnus lift on a rotating sphere at around the critical Reynolds number, Phys. Fluids 24, No. 014102 (2012).

30. Kim, J., Moin, P.: Application of a fractional-step method to incompressible NavierStokes equations. J. Comput. Phys. 59, 308-323 (1985).

31. Amsden, A.A., Harlow, F.H.: A simplified MAC technique for incompressible fluid flow calculations. J. Comput. Phys. 6, 322-325 (1970).

32. Rhie, C.M., Chow, W.L.: Numerical study of the turbulent flow past an airfoil with trailing edge separation. AIAA J. 21, 1525-1532 (1983).

33. Abe, K., Kondoh, T., Nagano, Y.: On Reynolds stress expressions and near-wall scaling parameters for predicting wall and homogeneous turbulent shear flows, Int. J. Heat Fluid Flow 18, 266-282 (1997)

34. Froehlich, J., Mellen, C.P., Rodi, W., Temmerman, L., Leschziner, M.A.: Highly resolved large-eddy simulation of separated flow in a channel with streamwise periodic constrictions, J. Fluid Mech. 526, 19-66 (2005). 